\title{
WIENER-HOPF FACTORIZATION FOR LÉVY PROCESSES HAVING POSITIVE JUMPS WITH RATIONAL TRANSFORMS
}

\author{
ALAN L. LEWIS, ${ }^{*}$ optioncity.net \\ ERNESTO MORDECKI,** Universidad de la República
}

\begin{abstract}
We show that the positive Wiener-Hopf factor of a Lévy process with positive jumps having a rational Fourier transform is a rational function itself, expressed in terms of the parameters of the jump distribution and the roots of an associated equation. Based on this, we give the closed form of the ruin probability for a Lévy process, with completely arbitrary negatively distributed jumps, and finite intensity positive jumps with a distribution characterized by a rational Fourier transform. We also obtain results for the ladder process and its Laplace exponent. A key role is played by the analytic properties of the characteristic exponent of the process and by a Baxter-Donsker-type formula for the positive factor that we derive.
\end{abstract}

Keywords: Lévy process; Wiener-Hopf factorization; Baxter-Donsker formula; ruin probability; rational Laplace transform; ladder process

2000 Mathematics Subject Classification: Primary 60G51

Secondary $60 \mathrm{~J} 50$

\section{Introduction}

\subsection{Lévy processes with rational positive jumps}

Consider a Lévy process $X=\left\{X_{t}\right\}_{t \geq 0}$ defined on a probability space $(\Omega, \mathcal{F}, \mathrm{P})$. For general reference on the subject, we refer the reader to [5], [17], or [25].

In this paper we are interested in a Lévy process of the following type. Consider a density function of the form

$$
p(x)=\sum_{k=1}^{v} \sum_{j=1}^{n_{k}} c_{k j}\left(\alpha_{k}\right)^{j} \frac{x^{j-1}}{(j-1) !} \exp \left(-\alpha_{k} x\right), \quad x>0 .
$$

Here the parameters $c_{k j}$ and $\alpha_{k}$ can in principle take complex values, but if we order the $\alpha_{k}$ by their real parts then $\alpha_{1}$ must be a positive real, while the others may be complex with

$$
0<\alpha_{1}<\operatorname{Re}\left(\alpha_{2}\right) \leq \cdots \leq \operatorname{Re}\left(\alpha_{v}\right) .
$$

This is the general form of the density of a random variable whose Fourier (or Laplace) transform is a rational function (i.e. the quotient of two polynomials):

$$
\hat{p}(u)=\int_{0}^{\infty} \mathrm{e}^{\mathrm{i} u x} p(x) \mathrm{d} x=\sum_{k=1}^{v} \sum_{j=1}^{n_{k}} c_{k j}\left(\frac{\mathrm{i} \alpha_{k}}{u+\mathrm{i} \alpha_{k}}\right)^{j} .
$$

Received 8 March 2007; revision received 21 November 2007.

* Postal address: 983 Bayside Cove, Newport Beach, CA 92660, USA. Email address: alewis@ optioncity.net

** Postal address: Facultad de Ciencias, Centro de Matemática, Universidad de la República, Iguá 4225, CP 11400

Montevideo, Uruguay. Email address: mordecki@cmat.edu.uy 
Assume that $X$ is a Lévy process with jump measure given by

$$
\Pi_{X}(\mathrm{~d} x)= \begin{cases}\pi^{+}(\mathrm{d} x)=\lambda p(x) \mathrm{d} x, & x>0 \\ \pi^{-}(\mathrm{d} x), & x<0\end{cases}
$$

where $\pi^{-}(\mathrm{d} x)$ is an arbitrary Lévy measure concentrated on the set $(-\infty, 0)$, describing the behavior of the negative jumps of the process. The positive jumps of the process have finite intensity $\lambda>0$ and magnitude distributed according to the probability density $p(x)$ given in (1.1).

We now describe the characteristic exponent $\psi$ of our model defined, for $u \in \mathbb{R}$, by the Lévy-Khinchine formula $\mathrm{E}\left(\exp \left(\mathrm{i} u X_{t}\right)\right)=\mathrm{e}^{t \psi(u)}$. Consider two independent Lévy processes $X^{-}$and $X^{+}$with respective characteristic exponents

$$
\begin{gathered}
\psi^{-}(u)=\mathrm{i} a u-\frac{1}{2} \sigma^{2} u^{2}+\int_{-\infty}^{0}\left(\mathrm{e}^{\mathrm{i} u x}-1-\mathrm{i} u h(x)\right) \pi^{-}(\mathrm{d} x), \\
\psi^{+}(u)=\lambda(\hat{p}(u)-1) .
\end{gathered}
$$

Here $a \in \mathbb{R}, \sigma \geq 0, h(x)=x \mathbf{1}_{\{|x| \leq 1\}}$ is a fixed truncation function, and $\hat{p}$ is as given in (1.2).

Our process can then be constructed as $X=X^{-}+X^{+}$and, consequently, has a characteristic exponent given by

$$
\psi(u)=\psi^{-}(u)+\psi^{+}(u) .
$$

In the actuarial literature, $X$ is the perturbed model obtained from the compound Poisson process $X^{+}$perturbed with the spectrally negative Lévy process $X^{-}$.

The goal of this paper is to establish the largest possible class of Lévy processes under which the double product form of the Laplace transform that we obtain in (2.2), below, holds for the distribution of the maximum defined in (1.7), below.

The presented results are similar to the classical results for random walks, where it is known that the computation of the law of the maximum requires only the specification of the positive jump structure of the processes, as exposed in [10, Chapter XII] and [14], or more recently in [1, Chapter VIII].

For Lévy processes, we generalize the results of [20], where the distribution of the maximum was considered when the Lévy measure had arbitrary negative part and positive jumps according to a mixture of exponential random variables, and [2] and [19], where positive phase-type distributed jumps were considered. In a recent paper by Pistorius [22] further results were obtained, in particular an algorithm for the computation of the parameters of the distribution of the maximum (that is of phase type) and a probabilistic representation of the ladder process. Among other works that study different aspects of ruin probabilities for Lévy processes, we mention [15], where asymptotic results for overshoots were obtained, [11], where a convolution formula was obtained for a classical claim process perturbed by an $\alpha$-stable Lévy process, and [13], where a Pollachek-Khinchine-type formula for the maximum of a Lévy process, constructed as the perturbation of a classical ruin process with a subordinator, was obtained.

In the present paper we pursue two objectives: the determination of the closed form of the distribution of the maximum for a Lévy process with positive jumps having a rational transform (this formally generalizes the class of phase-type distributions) and, at the same time, we are interested in the application of the classical complex variable methods to Lévy processes as in [3]. The obtained results have relevant applications in mathematical finance, where the determination of the Wiener-Hopf positive and negative factors give the solution of the optimal 
stopping problem related to the pricing of a perpetual call and put option, respectively, in a market model generated by a Lévy process (see [7] and [18]).

\subsection{Analytic properties and the Wiener-Hopf factorization}

The characteristic exponent $\psi^{-}$defined in (1.3) admits an analytic continuation to the half complex plane $\operatorname{Im}(z)<0$, continuous in $\operatorname{Im}(z) \leq 0$. The characteristic exponent $\psi^{+}$defined in (1.4) admits an analytic continuation to the half complex plane $\operatorname{Im}(z)>-\alpha_{1}$. In conclusion, the characteristic exponent (1.5) of the full model admits an analytic continuation to the complex strip $-\alpha_{1}<\operatorname{Im}(z)<0$, continuous in $-\alpha_{1}<\operatorname{Im}(z) \leq 0$, under the formula

$$
\psi(z)=\mathrm{i} a z-\frac{1}{2} \sigma^{2} z^{2}+\int_{-\infty}^{0}\left(\mathrm{e}^{\mathrm{i} z x}-1-\mathrm{i} z h(x)\right) \pi^{-}(\mathrm{d} x)+\lambda(\hat{p}(z)-1),
$$

which satisfies $\mathrm{E}\left(\exp \left(\mathrm{i} z X_{t}\right)\right)=\mathrm{e}^{t \psi(z)}$, by [25, Theorem 25.17]. Furthermore, $\psi(z)$ is a meromorphic function in the set $\operatorname{Im}(z)<0$, with poles in $-\mathrm{i} \alpha_{1}, \ldots,-\mathrm{i} \alpha_{v}$ and respective multiplicities $n_{1}, \ldots, n_{v}$. This justifies the label pole count for the quantity $n=n_{1}+\cdots+n_{\nu}$.

Denote by $\tau(q)$ an exponential random variable with parameter $q>0$, independent of the process $X$, and, for $q=0$, let $\tau(0)=\infty$. Our main interest in this paper is the determination of the law of the following random variables:

$$
M_{q}=\sup _{0 \leq t<\tau(q)} X_{t} \quad \text { and } \quad I_{q}=\inf _{0 \leq t<\tau(q)} X_{t},
$$

called the supremum and the infimum of the process, respectively, killed at rate $q$ if $q>0$. For the case in which $q=0$, we always assume that

$$
\mathrm{E}\left(X_{1}\right)=a+\int_{-\infty}^{-1} x \pi^{-}(\mathrm{d} x)+\lambda \int_{0}^{\infty} x p(x) \mathrm{d} x<0
$$

(including the case in which $\left.\mathrm{E}\left(X_{1}\right)=-\infty\right)$, and, consequently, the process drifts to $-\infty$, i.e. $\mathrm{P}\left(\lim _{t \rightarrow \infty} X_{t}=-\infty\right)=1$. This amounts to saying that the random variable $M_{0}$ in (1.7) is proper, while the random variable $I_{0}$ in (1.7) degenerates, i.e. $\mathrm{P}\left(I_{0}=-\infty\right)=1$. Their respective characteristic functions are the positive Wiener-Hopf factor given by

$$
\phi_{q}^{+}(u)=\mathrm{E}\left(\exp \left(\mathrm{i} u M_{q}\right)\right), \quad q \geq 0,
$$

and the negative Wiener-Hopf factor given by

$$
\phi_{q}^{-}(u)=\mathrm{E}\left(\exp \left(\mathrm{i} u I_{q}\right)\right), \quad q>0 .
$$

We consider the two following cases.

Case $(S):-X^{-}$is a subordinator.

Case (NS): $-X^{-}$is not a subordinator.

Lemma 1.1, below, is crucial in our development. (All proofs may be found in Section 3.)

Lemma 1.1. (Roots.) (a) Consider $q \geq 0$, and assume that $\mathrm{E}\left(X_{1}\right)<0$ for the case in which $q=0$. Then, the equation $q-\psi(z)=0$ has a simple purely imaginary root $-\mathrm{i} \beta_{1}(q)$, with $\beta_{1}(q)>0$, the unique root in the closure of the strip

$$
\mathcal{O}=\left\{z=u+\mathrm{i} v:-\beta_{1}(q)<v \leq 0\right\} .
$$


(b) For $q \geq 0$, the equation $q-\psi(z)=0$ has, in the set $\operatorname{Im}(z)<0$, a total of $\mu=\mu(q)$ distinct roots $-\mathrm{i} \beta_{1}(q),-\mathrm{i} \beta_{2}(q), \ldots,-\mathrm{i} \beta_{\mu}(q)$, with respective multiplicities $1, m_{2}(q), \ldots, m_{\mu}(q)$, ordered such that $0<\beta_{1}(q)<\operatorname{Re}\left(\beta_{2}(q)\right) \leq \cdots \leq \operatorname{Re}\left(\beta_{\mu}(q)\right)$. Furthermore, the total root count $m=1+m_{2}(q)+\cdots+m_{\mu}(q)$ does not depend on $q$ and is related to the pole count $n$ by the relation $m=n$ in case $(S)$ and $m=n+1$ in case (NS).

(c) Consider the polynomial

$$
B_{m, q}(u)=\prod_{j=1}^{\mu(q)}\left(\frac{u+\beta_{j}(q)}{\beta_{j}(q)}\right)^{m_{j}(q)} .
$$

Then, when $q$ tends to 0 the $q$-roots converge to the zero roots in such a way that

$$
B_{m, q}(u) \rightarrow B_{m, 0}(u), \quad q \rightarrow 0 .
$$

We will frequently omit the explicit display of $q$-dependencies in the roots. Consider the random variable $X_{\tau(q)}$, the process stopped at the independent exponential time $\tau(q)$. For $z \in \mathcal{O}$, defined in (1.8), using the Frullani integral to pass from (1.10), below, to (1.11), below, we have

$$
\begin{aligned}
\operatorname{Eexp}\left(\mathrm{i} z X_{\tau(q)}\right) & =\int_{0}^{\infty} q \mathrm{e}^{-q t} \mathrm{e}^{t \psi(z)} \mathrm{d} t \\
& =\frac{q}{q-\psi(z)} \\
& =\exp \int_{0}^{\infty} \frac{\mathrm{e}^{-q t}}{t}\left(\mathrm{e}^{t \psi(z)}-1\right) \mathrm{d} t \\
& =\exp \int_{0}^{\infty} \frac{\mathrm{e}^{-q t}}{t} \int_{-\infty}^{\infty}\left(\mathrm{e}^{\mathrm{i} z x}-1\right) \mathrm{P}\left(X_{t} \in \mathrm{d} x\right) \mathrm{d} t \\
& =\exp \int_{-\infty}^{\infty}\left(\mathrm{e}^{\mathrm{i} z x}-1\right)\left(\int_{0}^{\infty} \frac{\mathrm{e}^{-q t}}{t} \mathrm{P}\left(X_{t} \in \mathrm{d} x\right) \mathrm{d} t\right) \\
& =\exp \left(\int_{0}^{\infty}\left(\mathrm{e}^{\mathrm{i} z x}-1\right) \pi_{M}(\mathrm{~d} x)+\int_{-\infty}^{0}\left(\mathrm{e}^{\mathrm{i} z x}-1\right) \pi_{I}(\mathrm{~d} x)\right) \\
& =\exp \left(\psi_{M}(z)+\psi_{I}(z)\right),
\end{aligned}
$$

where, for $z \in \mathcal{O}$, we introduced the following notation:

$$
\begin{aligned}
& \psi_{M}(z)=\int_{0}^{\infty}\left(\mathrm{e}^{\mathrm{i} z x}-1\right) \pi_{M}(\mathrm{~d} x), \\
& \psi_{I}(z)=\int_{-\infty}^{0}\left(\mathrm{e}^{\mathrm{i} z x}-1\right) \pi_{I}(\mathrm{~d} x),
\end{aligned}
$$

with

$$
\pi_{M}(\mathrm{~d} x)=\int_{0}^{\infty} \frac{\mathrm{e}^{-q t}}{t} \mathrm{P}\left(X_{t} \in \mathrm{d} x\right) \mathrm{d} t, \quad \pi_{I}(\mathrm{~d} x)=\int_{-\infty}^{0} \frac{\mathrm{e}^{-q t}}{t} \mathrm{P}\left(X_{t} \in \mathrm{d} x\right) \mathrm{d} t .
$$

(This development is adapted from [5, Lemma VI.2.7], where it can be found for the case in which $z=u \in \mathbb{R}$. We rely on the argument there to interchange the integration orders to obtain (1.12).) Rogozin [24] established

$$
\phi_{q}^{+}(u)=\mathrm{E}\left(\exp \left(\mathrm{i} u M_{q}\right)\right)=\exp \left(\psi_{M}(u)\right), \quad \phi_{q}^{-}(u)=\mathrm{E}\left(\exp \left(\mathrm{i} u I_{q}\right)\right)=\exp \left(\psi_{I}(u)\right),
$$


and the Wiener-Hopf-Rogozin factorization:

$$
\frac{q}{q-\psi(u)}=\phi_{q}^{+}(u) \phi_{q}^{-}(u)=\exp \left(\psi_{M}(u)+\psi_{I}(u)\right) .
$$

In fact, our development (1.13) shows that this equality holds in $\mathcal{O}$, under the respective analytic continuations defined in (1.6), (1.14), and (1.15).

Furthermore, as the integral in (1.14) is also convergent when $\operatorname{Im}(z) \geq 0$, the exponent $\psi_{M}$ has an analytic continuation to the half-plane $\operatorname{Im}(z)>-\beta_{1}$ under the given formula, and, again by [25, Theorem 25.17], we have

$$
\operatorname{Eexp}\left(\mathrm{i} z M_{q}\right)=\exp \left(\psi_{M}(z)\right), \quad \operatorname{Im}(z)>-\beta_{1} .
$$

\section{Main results}

As the characteristic exponent $\psi_{M}$ of the maximum admits an analytic continuation to the strip of the complex plane $\operatorname{Im}(z)>-\beta_{1}$, the following Baxter-Donsker-type formula in (2.1), below, holds for the positive Wiener-Hopf factor. Note that (2.1) avoids integration at $z=0$.

Theorem 2.1. The positive Wiener-Hopffactor for a Lévy process with characteristic exponent given in (1.5) for $u>0$ satisfies

$$
\phi^{+}(\mathrm{i} u)=\mathrm{E}\left(\exp \left(-u M_{q}\right)\right)=\exp \left(\frac{1}{2 \pi \mathrm{i}} \int_{-\mathrm{i} v-\infty}^{-\mathrm{i} v+\infty} \frac{\mathrm{i} u}{z(z-\mathrm{i} u)} \log \left(\frac{q}{q-\psi(z)}\right) \mathrm{d} z\right)
$$

for any $v \in\left(0, \beta_{1}\right)$.

Remark 2.1. From the proof of Theorem 2.1, it can be seen that the rational structure of the positive jumps in (1.2) is not used. Consequently, (2.1) remains true under the condition of the existence of a strip of analyticity of the form $-\beta<\operatorname{Im}(z)<0$ for some $\beta>0$.

Based on the previous result, we can explicitly compute the positive factor in our model, showing that it has a rational transform.

Theorem 2.2. Consider a Lévy process $X$ with characteristic exponent given by (1.5) and $q \geq 0$. Assume that $\mathrm{E} X_{1}<0$ for the case in which $q=0$. Then the maximum $M_{q}$ has rational Laplace transform given by

$$
\phi_{q}^{+}(\mathrm{i} u)=\mathrm{E}\left(\exp \left(-u M_{q}\right)\right)=\prod_{k=1}^{v}\left(\frac{u+\alpha_{k}}{\alpha_{k}}\right)^{n_{k}} \prod_{j=1}^{\mu(q)}\left(\frac{\beta_{j}(q)}{u+\beta_{j}(q)}\right)^{m_{j}(q)} .
$$

Throughout the paper, we use the convention that in the absence of positive jumps $v=0$ and $\prod_{k=1}^{v}(\ldots)=1$ for every displayed product argument.

In order to obtain the density of the maximum $M_{q}$, we first denote

$$
\phi_{q}^{+}(\mathrm{i} u)=\mathrm{E}\left(\exp \left(-u M_{q}\right)\right)=\frac{A_{n}(u)}{B_{m, q}(u)},
$$

where $B_{m, q}$ is as defined in (1.9) and

$$
A_{n}(u)=\prod_{k=1}^{v}\left(\frac{u+\alpha_{k}}{\alpha_{k}}\right)^{n_{k}}
$$


Then we must invert the Laplace transform of the quotient of polynomials obtained above. The fractional expansion theorem states that

$$
\frac{A_{n}(u)}{B_{m}(u)}=d_{0}+d_{1} \frac{\beta_{1}}{u+\beta_{1}}+\sum_{k=2}^{\mu} \sum_{j=1}^{m_{k}} d_{k j}\left(\frac{\beta_{k}}{u+\beta_{k}}\right)^{j}
$$

where the first two coefficients are given by

$$
\begin{aligned}
d_{0} & = \begin{cases}0 & \text { in case (NS), } \\
\prod_{j=1}^{\mu}\left(\beta_{j}\right)^{m_{j}} \prod_{k=1}^{v}\left(\alpha_{k}\right)^{-n_{k}} & \text { in case (S), }\end{cases} \\
d_{1} & =\lim _{u \downarrow-\beta_{1}}\left(\frac{u+\beta_{1}}{\beta_{1}}\right) \phi_{q}^{+}(\mathrm{i} u) \\
& =\prod_{j=1}^{v}\left(\frac{\alpha_{j}-\beta_{1}}{\alpha_{j}}\right)^{n_{j}} \prod_{k=2}^{\mu}\left(\frac{\beta_{k}}{\beta_{k}-\beta_{1}}\right)^{m_{k}},
\end{aligned}
$$

and the rest of the coefficients are given by

$$
d_{k, m_{k}-j}=\frac{1}{j !\left(\beta_{k}\right)^{m_{k}-j}}\left[\frac{\partial^{j}}{\partial u^{j}}\left(\frac{A_{n}(u)}{B_{m}(u)}\left(u+\beta_{k}\right)^{m_{k}}\right)\right]_{u=-\beta_{k}}
$$

for $k=2, \ldots, \mu$ and $j=0, \ldots, m_{k}-1$. Thus, we have obtained the following result.

Corollary 2.1. Consider a Lévy process $X$ with characteristic exponent given by (1.5) and $q \geq 0$. Assume that $\mathrm{E} X_{1}<0$ for the case in which $q=0$. Then the random variable $M_{q}$ has a (generalized) density given by

$$
f_{M_{q}}(x)=d_{0} \delta_{0}(\mathrm{~d} x)+d_{1} \beta_{1} \exp \left(-\beta_{1} x\right)+\sum_{k=2}^{\mu} \sum_{j=1}^{m_{k}} d_{j k}\left(\beta_{k}\right)^{j} \frac{x^{j-1}}{(j-1) !} \exp \left(-\beta_{k} x\right),
$$

where $\delta_{0}(\mathrm{~d} x)$ is the Dirac delta at $x=0, \beta_{1}, \ldots, \beta_{\mu}$ are as given in Lemma 1.1, and the coefficients $d_{0}, d_{1}$, and $d_{j k}$ are given in (2.3), (2.4), and (2.5), respectively.

Our results can also be interpreted in terms of the ruin probabilities for the dual process $\hat{X}=\left\{\hat{X}_{t}=-X_{t}\right\}_{t \geq 0}$ for the case in which $q=0$. More precisely, if we define the ruin probability by

$$
R(x)=\mathrm{P}\left(\text { there exists } t \geq 0 \text { such that } x+\hat{X}_{t} \leq 0\right),
$$

from Corollary 2.1 we obtain the explicit formula

$$
R(x)=\mathrm{P}\left(\text { there exists } t \geq 0 \text { such that } x-X_{t} \leq 0\right)=\mathrm{P}\left(M_{0} \geq x\right)=\int_{x}^{\infty} f_{M_{0}}(y) \mathrm{d} y,
$$

with $f_{M_{0}}$ given in (2.6). 


\subsection{Ladder process}

From (2.2), under case (NS), we now derive the characteristic Lévy exponent $\kappa(q, u)$ of the ladder process $\ell=\left(L^{-1}, H\right)$ defined as in [5, p. 157]. With $R_{0}=0$, where $R_{0}=\inf \{t>$ $\left.0: X_{t}>0\right\}$, then $\operatorname{Eexp}\left(-q L_{t}^{-1}-u H_{t}\right)=\mathrm{e}^{-t \kappa(q, u)}$, where $\left\{L_{t}^{-1}, H_{t}\right\}$ is a two-dimensional subordinator for $t<\zeta \leq \infty$ with lifetime $\zeta$. For $t \geq \zeta$, both components are sent to $\infty$, which serves as a cemetery state. With these conventions, we can write

$$
\kappa(q, u)=\hat{k}+d_{1} q+d_{2} u+\int_{(0, \infty)^{2}}\left(1-\mathrm{e}^{-(q x+u y)}\right) \Pi_{\ell}(\mathrm{d} x, \mathrm{~d} y), \quad(q, u) \geq 0,
$$

where $\hat{k} \geq 0$ is the killing rate, the $\left\{d_{i}\right\}$ are nonnegative drift coefficients, and $\Pi_{\ell}(\mathrm{d} x, \mathrm{~d} y)$ is a Lévy measure supported on the set $(0, \infty) \times(0, \infty)$ which satisfies the condition

$$
\int \min \left[\sqrt{x^{2}+y^{2}}, 1\right] \Pi_{\ell}(\mathrm{d} x, \mathrm{~d} y)<\infty .
$$

Now divide both sides of (2.7) by $u$, and let $u$ tend to $\infty$. Then, for $u \geq \max \left[\frac{1}{2}, q\right]$, $\left(1-\mathrm{e}^{-(q x+u y)}\right) / u \leq 2 \min \left[\sqrt{x^{2}+y^{2}}, 1\right]$, and so the integral term vanishes in the limit by dominated convergence. This yields $\lim _{u \rightarrow \infty} \kappa(q, u) / u=d_{2}$. Taking the same limit in the formula $\phi_{q}^{+}(\mathrm{i} u)=\kappa(q, 0) / \kappa(q, u)$, we find that, for case (NS),

$$
\kappa(q, 0)=d_{2} \frac{N_{q}}{D}, \quad \text { where } \frac{N_{q}}{D}=\frac{\prod_{j=1}^{\mu(q)}\left(\beta_{j}(q)\right)^{m_{j}(q)}}{\prod_{k=1}^{v}\left(\alpha_{k}\right)^{n_{k}}},
$$

with an obvious definition of $N_{q}$ and $D$. For $q>0, D>0$, since $p(x) \geq 0$. Also, $N_{q}>0$, since all the other terms are positive.

Not all ladder height processes $\left\{H_{t}\right\}$ have a positive drift; however, $\left\{H_{t}\right\}$ is known to have a strictly positive drift if and only if the Lévy process $X$ can 'creep' upwards across any level $x>0$ (cf. [23] for a discussion). Necessary and sufficient conditions for upwards creep have been found in [26]. However, we only need the sufficient conditions as given in [4]: upwards creep occurs if (i) $\sigma>0$, (ii) $X$ has bounded variation and positive drift coefficient, or (iii) $\int_{-1}^{0} x \Pi_{X}(\mathrm{~d} x)=-\infty$ and $\int_{0}^{1} x \Pi_{X}(\mathrm{~d} x)<\infty$. In our class of models, case (NS) implies that we have $\sigma>0$, a positive drift coefficient, or $\int_{-1}^{0} x \Pi_{X}(\mathrm{~d} x)=-\infty$. Since our upwards jump process has finite intensity, at least one sufficiency condition holds. Now assured that $d_{2}>0$, we can (and now do) adopt a normalization of the local time such that $d_{2}=1$. We then conclude from $\kappa(q, u)=\kappa(q, 0) / \phi_{q}^{+}(\mathrm{i} u)$ that, for case (NS),

$$
\kappa(q, u)=\frac{\prod_{j=1}^{\mu(q)}\left(u+\beta_{j}(q)\right)^{m_{j}(q)}}{\prod_{k=1}^{v}\left(u+\alpha_{k}\right)^{n_{k}}}, \quad(q, u) \geq 0 .
$$

Equation (2.8) is our main result for this section. Holding $q \geq 0$ fixed, (2.8) provides a meromorphic extension of $\kappa(q, u)$ to the entire complex $u$-plane. From it, we immediately obtain the killing rate

$$
\hat{k}=\kappa(0,0)=\frac{\prod_{j=1}^{\mu(0)}\left(\beta_{j}(0)\right)^{m_{j}(0)}}{\prod_{k=1}^{v}\left(\alpha_{k}\right)^{n_{k}}} .
$$

Under our standing requirement that $\mathrm{E} X_{1}<0$ for Lévy processes $X$ that are not killed, there exists a nontrivial solution $\beta_{1}(0)>0$ and the numerator in (2.9) is strictly positive. This class 
of processes drift to $-\infty$, have a last maximum with probability 1 , and a strictly positive killing rate for the ladder process. (In the alternative, with $\mathrm{E} X_{1}>0, \lim _{q \downarrow 0} \beta_{1}(q)=0$. This means that $\hat{k}=0$ as expected, since there is no last ladder epoch.)

Now, let us compare (2.8) with known results for the so-called spectrally one-sided Lévy process, i.e. a drift and diffusion component may be present, but the jumps are entirely positive or negative.

If there are no positive jumps then the pole count $v$ equals 0 from Lemma 1.1. For case (NS), the root count is one larger, so $\mu(q)=1$. Hence, (2.8) reads $\kappa(q, u)=\beta_{1}(q)+u$, where $\beta_{1}(q)$ is the sole nonnegative solution to $\psi(-\mathrm{i} u)=q$ with $(q, u)>0$. This is correct; see [5, Section VII $(2)]$, where $\beta_{1}(q)$ is denoted as $\Phi(q)$.

If there are no negative jumps (NNJ), let $\beta_{0}(q)$ be the sole nonnegative solution to $\psi(\mathrm{i} u)=q$ with $(q, u)>0$. With the set $\left\{\beta_{j}(q): 1 \leq j \leq \mu(q)\right\}$ previously defined, restricting our class of models to NNJ means that

$$
\psi(\mathrm{i} u)-q=\frac{1}{2} \sigma^{2} u^{2}-a u+\lambda(\hat{p}(\mathrm{i} u)-1)-q=\left(\frac{\sigma^{2}}{2}\right) \frac{\prod_{j=0}^{\mu(q)}\left(u+\beta_{j}(q)\right)^{m_{j}(q)}}{\prod_{k=1}^{v}\left(u+\alpha_{k}\right)^{n_{k}}}
$$

with $m_{0}(q)=1$. The right-hand side of (2.10) expresses the left-hand side as a rational function of $u$, matching the roots, poles, multiplicities, and behavior as $u$ tends to $\infty$. With NNJ, we insist that $\sigma^{2}>0$ because $\sigma^{2}=0$ would force the choice $a>0$ to preserve case (NS). But the combination of $\sigma^{2}=0, a>0$, and NNJ is inconsistent with our standing condition $\mathrm{E} X_{1}<0$. Finally, we appeal to (2.8) to be valid throughout the complex $u$-plane with the exception of the isolated poles. Appending $u+\beta_{0}(q)$ to both products in (2.8), and using (2.10), yields

$$
\kappa(q, u)=\frac{\prod_{j=0}^{\mu(q)}\left(u+\beta_{j}(q)\right)^{m_{j}(q)}}{\left(u+\beta_{0}(q)\right) \prod_{k=1}^{v}\left(u+\alpha_{k}\right)^{n_{k}}}=\frac{2}{\sigma^{2}}\left(\frac{\Psi(u)-q}{u+\beta_{0}(q)}\right),
$$

using the Laplace exponent $\Psi(u) \equiv \psi(\mathrm{i} u)$. This is also correct; see, for example, [13, Equation (5.4)] or make an easy independent calculation from Fristedt's formula for $\kappa(q, u)$ [5, Corollary VI.10].

In principle, the ladder process jump measure $\Pi_{\ell}(\mathrm{d} x, \mathrm{~d} y)$ can be obtained by the inversion of (2.7). While this may not be easy, consider the simpler case of the ladder height jump measure $\Pi_{H}(\mathrm{~d} y)$, where (2.7) reads as $\kappa(0, u)=\hat{k}+u+\int\left(1-\mathrm{e}^{-u y}\right) \Pi_{H}(\mathrm{~d} y)$. Using (2.8) and (2.9), and letting $u$ tend to $\infty$, we find that $\Pi_{H}(0, \infty)<\infty$. In other words, prior to being killed, $\left\{H_{t}\right\}$ is a Poisson process plus a unit drift with jump intensity

$$
\begin{aligned}
\lambda_{H} & \equiv \Pi_{H}(0, \infty) \\
& =\lim _{u \rightarrow \infty}\left\{\frac{\prod_{j=1}^{\mu(0)}\left(u+\beta_{j}(0)\right)^{m_{j}(0)}}{\prod_{k=1}^{v}\left(u+\alpha_{k}\right)^{n_{k}}}-u-\frac{\prod_{j=1}^{\mu(0)}\left(\beta_{j}(0)\right)^{m_{j}(0)}}{\prod_{k=1}^{v}\left(\alpha_{k}\right)^{n_{k}}}\right\} \\
& =\sum_{j=1}^{\mu(0)} m_{j}(0) \beta_{j}(0)-\sum_{k=1}^{v} n_{k} \alpha_{k}-\frac{\prod_{j=1}^{\mu(0)}\left(\beta_{j}(0)\right)^{m_{j}(0)}}{\prod_{k=1}^{v}\left(\alpha_{k}\right)^{n_{k}}} .
\end{aligned}
$$

This leaves $\int \mathrm{e}^{-u y} \Pi_{H}(\mathrm{~d} y)=\hat{k}+u+\lambda_{H}-\kappa(0, u)$. The right-hand side of the last equality is a rational function of $u$ with poles at $u=-\alpha_{k}, k=1, \ldots, v$, pole multiplicities $n_{k}$, and decaying as $O(1 / u)$ as $u$ tends to $\infty$. But this also describes the transform $\hat{p}(i u)$ of (1.2) expressed as a rational function. Hence, by inversion, we see that the ladder height jump 
distribution has essentially the same structure as the distribution of the positive jumps of the underlying $X$-process, although the two intensities $\left(\lambda, \lambda_{H}\right)$ differ. It is also easy to see from (2.11), recalling the discussion above for no positive jumps, that $\lambda_{H}$ vanishes if $\lambda$ vanishes.

Similar work for the case of phase-type positive jumps can be found in [22].

\subsection{Asymptotic behavior for general Lévy processes}

It is interesting to note that the second term $d_{1} \beta_{1} \exp \left(-\beta_{1} x\right)$ in (2.6) determines the asymptotic behavior of the density of the maximum for large values of $x$ in our model (1.5). For the case in which $q=0$, this asymptotic behavior for a general Lévy process whose characteristic exponent has a purely imaginary root $-\mathrm{i} \beta, \beta>0$, was obtained in [6] and satisfies

$$
\lim _{x \rightarrow \infty} \mathrm{P}\left(M_{0}>x\right) \mathrm{e}^{\beta x}=C .
$$

The constant $C$ given in [6] is difficult to calculate. In our model with characteristic exponent defined by (1.5) the constant $C=d_{1}$. The following result gives the constant in (2.12) for general Lévy models. The proof can be found in Section 3.

Proposition 2.1. Consider a general Lévy process with characteristic exponent $\psi(u)$. Assume that there exists a positive $\beta$ such that $\psi(-\mathrm{i} \beta)=0$. Then the constant in (2.12) is determined by

$$
C=\lim _{u \downarrow-\beta}\left(\frac{u+\beta}{\beta}\right) \phi_{0}^{+}(\mathrm{i} u) .
$$

To complete the story, we sketch how our general expression (2.13) easily generates known results for $C$ in spectrally one-sided Lévy models. In both cases we assume that the drift $\mu \equiv \mathrm{E} X_{1}<0$ and, for simplicity, that the constant $C$ is not 0 or $\infty$.

First consider the spectrally negative case. Since the process always attains new maxima in a continuous manner, form the well-known martingale $\xi_{t}=\exp \left(\mathrm{i} z X_{t}-t \psi(z)\right)$, real and analytic for $z$ on the negative imaginary axis, where we work throughout the remainder of this section. Stopping this martingale at $t=\tau_{x}$, the first hitting time of $x \geq 0$ (justifiable by a limiting argument with Doob's optional stopping theorem) implies that $1=$ $\mathrm{e}^{\mathrm{i} z x} \mathrm{E}\left(\exp \left(-\tau_{x} \psi(z)\right) \mathbf{1}_{\left\{\tau_{x}<\infty\right\}}\right)$. Choose $z=-\mathrm{i} \beta$, where $\psi(-\mathrm{i} \beta)=0$ and $\beta>0$ by virtue of $\mathrm{E} X_{1}<0$. Then $1=\mathrm{e}^{\beta x} \mathrm{E}\left(\mathbf{1}_{\left\{\tau_{x}<\infty\right\}}\right)=\mathrm{e}^{\beta x} \mathrm{P}\left(M_{0} \geq x\right)$, so $C=1$, the known result. Since the distribution $\mathrm{P}\left(M_{0} \leq x\right)$ is nondefective, then $\phi_{0}^{+}(\mathrm{i} u)=\beta /(u+\beta)$, showing that (2.13) is correct, although not especially useful in this case.

More interesting is the spectrally positive case, where it is again notationally cleaner to use the Laplace exponent $\Psi(s)=\psi($ is $)$. Here $s$ is a real parameter, so that $\mathrm{E}\left(\exp \left(-s X_{t}\right)\right)=\mathrm{e}^{t \Psi(s)}$. It was originally proved, in [27] and transparently in [12], that

$$
\mathrm{E}\left(\exp \left(-s M_{0}\right)\right)=-\frac{\mu s}{\Psi(s)}, \quad s \geq 0 .
$$

Since, by assumption, $\psi(-\mathrm{i} \beta)=0$ for some $\beta>0$, then (2.14) can be extended to $-\beta<s<\infty$. We can then apply (2.14) to (2.13). Moreover, because the limit $C$ in (2.13) exists and is not 0 , then the 0 of $\Psi(s)$ at $s=-\beta$ must be simple. This allows us to invoke L'Hospital's rule and immediately find that

$$
C=-\mu \lim _{s \downarrow-\beta}\left(\frac{s+\beta}{\beta}\right) \frac{s}{\Psi(s)}=\frac{\mu}{\Psi^{\prime}(-\beta)}=\frac{-\Psi^{\prime}(0)}{\Psi^{\prime}(-\beta)}=\frac{|\mu|}{\mu^{*}},
$$


where $\mu^{*}$ is defined to be the drift in the associated process $\Psi^{*}(s) \equiv \Psi(s-\beta)$. The result that $C=|\mu| / \mu^{*}$ for a spectrally positive Lévy process (such that $\mu<0$ and an associated process exists with $\mu^{*}>0$ ) was established in [9] as a consequence of a much lengthier argument.

\section{Proofs}

Proof of Lemma 1.1. (a) Consider an auxiliary function $a(v)=\psi(\mathrm{i} v)$, defined for $v \in$ $\left(-\alpha_{1}, 0\right]$, that satisfies

(i) $a(v)$ is a real convex analytic function in $\left(-\alpha_{1}, 0\right)$ with $a(0)=0$;

(ii) $a^{\prime}(0-)=-\mathrm{E}\left(X_{1}\right)>0$ for the case in which $q=0$;

(iii) $\lim _{v \downarrow-\alpha_{1}} a(v)=\infty$.

For the case in which $q>0$, the continuity property (i) gives the existence of a negative root $-\beta_{1}$ of the equation $a(v)=q$. For the case in which $q=0$, properties (ii) and (iii) imply that the function $a(v)$ has an absolute minimum in a certain point $v_{0} \in\left(\alpha_{1}, 0\right)$ and that the equation $a(v)=0$ has a root $-\beta_{1}<v_{0}$. In both cases, as $a^{\prime}\left(-\beta_{1}\right)<0$, it is a simple root. Now, for $z=u+\mathrm{i} v$ in the $\operatorname{strip} \mathcal{O}$, the ridge property gives, for $z \in \mathcal{O}$,

$$
\operatorname{Re}(\psi(z)) \leq \psi(\mathrm{i} v)<q,
$$

giving $q-\psi(z) \neq 0$ in $\mathcal{O}$. It is also true that $\psi\left(u-\mathrm{i} \beta_{1}\right) \neq q$ if $u \neq 0$. In the case in which the equality $\psi\left(u-\mathrm{i} \beta_{1}\right)=q$ holds for some $u$, denoting by $F(\mathrm{~d} x)$ the distribution of $X_{1}$, we have

$$
\begin{aligned}
\int_{-\infty}^{\infty} \exp \left(\beta_{1} x\right) \mathrm{d} F(x) & =\exp \left(\psi\left(-\mathrm{i} \beta_{1}\right)\right) \\
& =\mathrm{e}^{q} \\
& =\exp \left(\psi\left(u-\mathrm{i} \beta_{1}\right)\right) \\
& =\int_{-\infty}^{\infty} \exp \left(\beta_{1} x\right) \cos (u x) \mathrm{d} F(x) .
\end{aligned}
$$

We conclude that $F(x)$ can concentrate mass only in the set $x=2 \pi k / u, k \in \mathbb{Z}$, i.e. $F(x)$ is a lattice random variable, a situation that is excluded by the form of the positive jumps of our process (that have positive probability of hitting any interval). This implies that $-\mathrm{i} \beta_{1}$ is the only root in the closure of $\mathcal{O}$. This concludes the proof of (a).

We first show part (b) for the case in which $p(x) \equiv 0$, i.e. when we do not have positive jumps. Then we have to prove that, in case (S), we have $q-\psi(z) \neq 0$ for $\operatorname{Im}(z)<0$ and, in case (NS), that $q-\psi(z)=0$ has exactly one root in the $\operatorname{set} \operatorname{Im}(z)<0$.

First consider case (S). As $-X^{-}$is a subordinator,

$$
\psi^{-}(z)=\mathrm{i} a z+\int_{-\infty}^{0}\left(\mathrm{e}^{\mathrm{i} z x}-1\right) \pi^{-}(\mathrm{d} x)
$$

for some $a \leq 0$. Let $z=u+\mathrm{i} v$ and observe that when $v=\operatorname{Im}(z)<0$, we have

$$
\operatorname{Re}\left(q-\psi^{-}(z)\right)=q+a v+\int_{-\infty}^{0}\left(1-\mathrm{e}^{-v x} \cos u x\right) \pi^{-}(\mathrm{d} x)>q .
$$

This means that $q-\psi^{-}(z)$ has no 0 s in $\operatorname{Im}(z)<0$, i.e. we have established that $m=0$ in case $(\mathrm{S})$. 
Now consider case (NS). When $-X^{-}$is not a subordinator, the equation $q-\psi^{-}(z)=0$ has exactly one purely imaginary root in the half-plane $\operatorname{Im}(z)<0$ if $q>0$ or if $q=0$ and $\mathrm{E}\left(X_{1}\right)<0$ (see [5, Section VII.1]). In conclusion, we have established that $m=1$ in case (NS).

To complete the proof of part (b), we consider that the full model (with positive jumps) is a perturbation of the model just considered, and we prove, with the help of Rouche's theorem, that the winding number $w=m-n$, i.e. the difference between the number of roots minus the pole count, remains constant when we add the jumps. To do this, let

$$
\begin{gathered}
f(z)=q+\lambda-\psi^{-}(z)=q+\lambda-\mathrm{i} a z+\frac{1}{2} \sigma^{2} z^{2}+\int_{-\infty}^{0}\left(1+\mathrm{i} z h(x)-\mathrm{e}^{\mathrm{i} z x}\right) \pi^{-}(\mathrm{d} x), \\
g(z)=-\lambda \hat{p}(z),
\end{gathered}
$$

and observe that $f=0$ is the equation considered previously (now with $q+\lambda$ instead of $q$ ), and $f+g=q-\psi(z)=0$ is the equation under consideration, the full model.

The idea is then to establish that $|g|<|f|$ on a contour of the form

$$
\left\{z=r \mathrm{e}^{\mathrm{i} \theta}, \pi \leq \theta \leq 2 \pi\right\} \cup\{\operatorname{Im}(z)=0, r \leq|z| \leq R\} \cup\left\{z=R \mathrm{e}^{\mathrm{i} \theta}, \pi \leq \theta \leq 2 \pi\right\}
$$

with $0 \leq r<R$ (small $r$ and large $R$ ), contains all the poles of $q-\psi$, in order to show that the winding numbers of $f$ and $f+g=q-\psi$ coincide. To verify that $|g|<|f|$ on (3.2), we proceed by steps.

Step 1. Assume that $q>0$. We prove that $|g|<|f|$ when $\operatorname{Im}(z)=0$ for both case (S) and case (NS). Let $z=u+\mathrm{i} v$, and observe that, owing to the fact that a distribution with rational transform is nonlattice (as it has density), we know that $\hat{p}(u) \neq 1$ (see [21, Lemma I.3.2]). So

$$
|g(u)|<\lambda \quad \text { if } u \neq 0 .
$$

On the other side,

$$
|f(u)| \geq \operatorname{Re}(f(u)) \geq f(0)=\lambda+q>\lambda,
$$

completing the proof for the case in which $r=0$.

Step 2. Assume that $q=0$. The preceding argument does not work for the case in which $u=0$, when $|g(0)|=\lambda$. But, in this case the condition

$$
\text { E } X_{1}=a+\int_{-\infty}^{-1} x \pi^{-}(\mathrm{d} x)+\lambda \int_{0}^{\infty} x p(x) \mathrm{d} x<0
$$

ensures that

$$
f^{\prime}(\mathrm{i} 0-)>g^{\prime}(\mathrm{i} 0-) \text {. }
$$

As the limit in the derivative is taken with $z \rightarrow 0$ and $\operatorname{Im}(z)<0$, this tells us that there exists a small enough $r>0$ such that the inequality $|f(z)|>|g(z)|$ holds for $z=r \mathrm{e}^{\mathrm{i} \theta}, \pi \leq \theta \leq 2 \pi$. This means that in this case the contour should have a modification of the form $z=r \mathrm{e}^{\mathrm{i} \theta}, \pi \leq$ $\theta \leq 2 \pi$, to exclude the point $z=0$.

Step 3. In the case in which $\sigma>0$ let us now verify that, for big enough $R$,

$$
|g(z)|<|f(z)| \quad \text { when } z=R \mathrm{e}^{\mathrm{i} \theta}, \pi \leq \theta \leq 2 \pi .
$$

To begin with, note that

$$
\lim _{|z| \rightarrow \infty} g(z)=-\lambda \lim _{|z| \rightarrow \infty} \hat{p}(z)=0
$$


As, by dominated convergence, we obtain

$$
\lim _{|z| \rightarrow \infty} \int_{-\infty}^{0} \frac{\mathrm{e}^{\mathrm{i} z x}-1-\mathrm{i} z h(x)}{|z|^{2}} \pi^{-}(\mathrm{d} x)=0,
$$

we conclude that $f(z)=-\left(\sigma^{2} / 2\right) z^{2}+o\left(|z|^{2}\right)$ when $\operatorname{Im}(z)<0$. Taking (3.4) into account, we obtain (3.3).

Step 4. Now let us consider the case in which $\sigma=0$. In case (S) (3.1) gives $|f|>\lambda$, which ensures that condition (3.3) holds. Now consider case (NS). In this situation we know that

$$
\int_{-1}^{0} x \pi^{-}(\mathrm{d} x)=-\infty
$$

This ensures the existence of $c \in(-1,0)$ such that

$$
a_{c}=a-\int_{-1}^{c} x \pi^{-}(\mathrm{d} x)>0 \text {. }
$$

Now consider

$$
\frac{\psi^{-}(z)}{\mathrm{i} z}=a_{c}+\int_{c}^{0} \frac{\mathrm{e}^{\mathrm{i} z x}-1-\mathrm{i} z x}{\mathrm{i} z} \pi^{-}(\mathrm{d} x)+\int_{-\infty}^{c} \frac{\mathrm{e}^{\mathrm{i} z x}-1}{\mathrm{i} z} \pi^{-}(\mathrm{d} x) .
$$

We will consider $|z| \rightarrow \infty$ with $\operatorname{Im}(z)<0$. It is clear that

$$
\lim _{|z| \rightarrow \infty}\left|\int_{-\infty}^{c} \frac{\mathrm{e}^{\mathrm{i} z x}-1}{\mathrm{i} z} \pi^{-}(\mathrm{d} x)\right| \leq \lim _{|z| \rightarrow \infty} \frac{2 \int_{-\infty}^{c} \pi^{-}(\mathrm{d} x)}{|z|}=0 .
$$

Furthermore, we check that, with $x<0$,

$$
\operatorname{Re}\left(\frac{1+\mathrm{i} z x-\mathrm{e}^{\mathrm{i} z x}}{\mathrm{i} z}\right)=\int_{x}^{0} \operatorname{Re}\left(\mathrm{e}^{\mathrm{i} z t}-1\right) \mathrm{d} t<0,
$$

to obtain

$$
\begin{aligned}
\frac{|f(z)|}{|z|} & \geq \operatorname{Re}\left(\frac{-f(z)}{\mathrm{i} z}\right) \\
& =a_{c}-\int_{0}^{c} \operatorname{Re}\left(\frac{1+\mathrm{i} z x-\mathrm{e}^{\mathrm{i} z x}}{\mathrm{i} z}\right) \pi^{-}(\mathrm{d} x)+o(1) \\
& \geq a_{c}+o(1)
\end{aligned}
$$

As $a_{c}>0$, in view of (3.4), we obtain $|g|<|f|$ over $R \mathrm{e}^{\mathrm{i} \theta}, 0 \leq \theta \leq \pi$, for big enough $R$. This concludes the proof of part (b).

In order to prove part (c), it is enough to verify that, for each $j=1, \ldots, n$, the equation $q-\psi(z)=0$ has $n_{j}$ roots, all converging to $\beta_{j}(0)$ as $q$ tends to 0 .

In order to do this, take arbitrarily small $\delta$ and consider the disk $D=D\left(\beta_{j}, \delta\right)$ with center $\beta_{j}$ and radius $\delta$. In particular, $\delta$ is small enough such that the equation $q-\psi(z)=0$ has the only root $\beta_{j}$ in the closure of $D$, and $D$ is contained in $\operatorname{Im}(z)>0$. Consequently, there exists $q_{0}>0$ such that

$$
q_{0}<\inf \{|\psi(z)|: z \in \partial D\} .
$$

From this and Rouche's theorem, we obtain $|-q|<|\psi(z)|$ on $\partial D$, and the number of roots of $q-\psi(z)$ and $\psi(z)$ on $D$ coincide. This concludes the proof of Lemma 1.1. 
Remark 3.1. As $a_{c}>0$ is arbitrary, the result in (3.5) implies that

$$
\lim _{\operatorname{Im}(z)<0,|z| \rightarrow \infty} \frac{\psi^{-}(z)}{|z|}=\infty .
$$

This fact was obtained in [8] for the case in which $\operatorname{Im}(z)=0$.

Proof of Theorem 2.1. We begin by taking the $\operatorname{logarithm}$ of $(1.16)$ when $\operatorname{Im}(z)=-v$, to obtain

$$
\log \frac{q}{q-\psi(z)}=\psi_{M}(z)+\psi_{I}(z)
$$

We show that

$$
\int_{-\mathrm{i} v-\infty}^{-\mathrm{i} v+\infty} \frac{\mathrm{i} u}{z(z-\mathrm{i} u)} \psi_{I}(z) \mathrm{d} z=0, \quad \int_{-\mathrm{i} v-\infty}^{-\mathrm{i} v+\infty} \frac{\mathrm{i} u}{z(z-\mathrm{i} u)} \psi_{M}(z) \mathrm{d} z=2 \pi \mathrm{i} \psi_{M}(\mathrm{i} u) .
$$

Observe that these two equalities, in view of (3.6), are equivalent to (2.1) and their verification concludes the proof of the theorem.

Consider then, for fixed $v \in\left(0, \beta_{1}\right)$, the line segment

$$
I_{R}=\{z=-\mathrm{i} v+u,|z| \leq R\},
$$

the arcs

$$
C_{R}^{+}=\{|z|=R, \operatorname{Im}(z) \geq-v\}, \quad C_{R}^{-}=\{|z|=R, \operatorname{Im}(z) \leq-v\},
$$

and the closed contours

$$
U_{R}=C_{R}^{+} \cup I_{R}, \quad L_{R}=C_{R}^{-} \cup I_{R}
$$

First observe that

$$
\oint_{L_{R}} \frac{\mathrm{i} u}{z(z-\mathrm{i} u)} \psi_{I}(z) \mathrm{d} z=0, \quad \oint_{U_{R}} \frac{\mathrm{i} u}{z(z-\mathrm{i} u)} \psi_{M}(z) \mathrm{d} z=2 \pi \mathrm{i} \psi_{M}(\mathrm{i} u) .
$$

The first statement holds because the integrand is analytic inside the closed integration contour, the second statement holds by the residue theorem. In the latter case the integrand has two poles inside the contour $U_{R}$. The first residue vanishes, as $\psi_{M}(0)=0$, and the second residue gives the result.

It remains to check that the integrals in both arcs vanish as $R$ tends to $\infty$. Observe that in these $\operatorname{arcs} z=R \mathrm{e}^{\mathrm{i} \theta}$ and $\mathrm{d} z=\mathrm{i} z \mathrm{~d} \theta$ for values of $\theta$ in the respective intervals $\theta \in \Theta_{R}^{+}$and $\theta \in \Theta_{R}^{-}$. For the first arc, we have

$$
\int_{C_{R}^{-}} \frac{\mathrm{i} u}{z(z-\mathrm{i} u)} \psi_{I}(z) \mathrm{d} z=\int_{\Theta_{R}^{-}} \mathrm{i} \mathrm{d} \theta \frac{\mathrm{i} u}{(z-\mathrm{i} u)} \int_{-\infty}^{0}\left(\mathrm{e}^{\mathrm{i} z x}-1\right) \pi_{I}(\mathrm{~d} x) .
$$

The integrand can be bounded by

$$
\frac{\left|\mathrm{e}^{\mathrm{i} z x}-1\right|}{|z-\mathrm{i} u|} \leq \frac{|z x| \wedge 2}{|z|} \leq|x| \wedge \frac{2}{R}
$$


for $|z|=R$ big enough. So,

$$
\begin{aligned}
\left|\int_{C_{R}^{-}} \frac{u}{(z-\mathrm{i} u)} \psi_{I}(z) \mathrm{d} z\right| & \leq \int_{\Theta_{R}^{-}} \mathrm{d} \theta \int_{-\infty}^{0}\left|\frac{u\left(\mathrm{e}^{\mathrm{i} z x}-1\right)}{z-\mathrm{i} u}\right| \pi_{I}(\mathrm{~d} x) \\
& \leq u \pi \int_{-\infty}^{0}\left(|x| \wedge \frac{2}{R}\right) \pi_{I}(\mathrm{~d} x) \\
& \rightarrow 0 \text { as } R \rightarrow \infty
\end{aligned}
$$

by dominated convergence. Observe that the bounds obtained in (3.8) justify the change of order in the integrals.

The integral in $C_{R}^{+}$is similar, the main difference being the bound of the integrand. As $|z|-u \leq|z-\mathrm{i} u|$, we obtain

$$
\frac{\left|\mathrm{e}^{\mathrm{i} z x}-1\right|}{|z-\mathrm{i} u|} \leq \frac{|z x| \wedge\left(\mathrm{e}^{v x}+1\right)}{|z|-u} \leq 2\left(|x| \wedge \frac{\mathrm{e}^{v x}+1}{R}\right)
$$

for $|z|=R$ big enough also. In this case the Lebesgue dominated convergence theorem works owing to the fact that $\int_{1}^{\infty} \mathrm{e}^{v x} \pi_{M}(\mathrm{~d} x)<\infty$, a condition which is equivalent to $\mathrm{E} \exp \left(v M_{q}\right)<$ $\infty$. This concludes the proof.

Proof of Theorem 2.2. First consider the case in which $q>0$. By Lemma 1.1 we know that the equation $q-\psi(z)=0$ has $\mu$ different roots $-\mathrm{i} \beta_{1},-\mathrm{i} \beta_{2}, \ldots,-\mathrm{i} \beta_{\mu}$ with respective multiplicities $1, m_{2}, \ldots, m_{\mu}$ and root count $m=1+m_{2}+\cdots+m_{\mu}$ equal to $n$ in case (S) and equal to $n+1$ in case (NS).

Let

$$
G_{q}^{+}(z)=\prod_{k=1}^{v}\left(\frac{z+\mathrm{i} \alpha_{k}}{\mathrm{i} \alpha_{k}}\right)^{n_{k}} \prod_{j=1}^{\mu}\left(\frac{\mathrm{i} \beta_{j}}{z+\mathrm{i} \beta_{j}}\right)^{m_{j}},
$$

and define $G_{q}^{-}(z)$ by the relation

$$
\frac{q}{q-\psi(z)}=G_{q}^{+}(z) G_{q}^{-}(z)
$$

As $G_{q}^{+}$is an infinitely divisible characteristic function with support on $[0, \infty)$, Rogozin's factorization (1.16) suggests that this is the correct factorization. If we knew that $G_{q}^{-}(z)$ is an infinitely divisible characteristic function with support on $(-\infty, 0]$, the uniqueness of the factorization in (1.16) would give the answer. As we do not have this information, we apply Theorem 2.1. From definition (3.9) we observe that

(i) $G_{q}^{-}(0)=1$;

(ii) $G_{q}^{-}(z)$ is a nonvanishing analytic function on the half-plane $\operatorname{Im}(z)<0$ and is continuous on $\operatorname{Im}(z) \leq 0$

(iii) $G_{q}^{-}(z)$ is a bounded function on the half-plane $\operatorname{Im}(z) \leq 0$, as follows from the proof of Lemma 1.1.

These properties ensure that both integrals

$$
I^{ \pm}(u)=\frac{1}{2 \pi \mathrm{i}} \int_{-\mathrm{i} v-\infty}^{-\mathrm{i} v+\infty} \frac{\mathrm{i} u}{z(z-\mathrm{i} u)} \log \left(G_{q}^{ \pm}(z)\right) \mathrm{d} z
$$


are convergent. Based on (3.9), we compute the positive Wiener-Hopf factor integral in Theorem 2.1 by

$$
\begin{aligned}
\phi^{+}(\mathrm{i} u) & =\mathrm{E}\left(\exp \left(-u M_{q}\right)\right) \\
& =\exp \left(\frac{1}{2 \pi \mathrm{i}} \int_{-\mathrm{i} v-\infty}^{-\mathrm{i} v+\infty} \frac{\mathrm{i} u}{z(z-\mathrm{i} u)} \log \left(\frac{q}{q-\psi(z)}\right) \mathrm{d} z\right) \\
& =\exp \left(I^{+}(u)+I^{-}(u)\right) .
\end{aligned}
$$

Equation (2.2) will then follow from the evaluation of the integrals

$$
I^{+}(u)=\log G^{+}(\mathrm{i} u), \quad I^{-}(u)=0 .
$$

Consider the contours $U_{R}$ and $L_{R}$ defined in (3.7). In order to obtain the first integral in (3.10), observe that

$$
\begin{aligned}
\frac{1}{2 \pi \mathrm{i}} \int_{U_{R}} \frac{\mathrm{i} u}{z(z-\mathrm{i} u)} \log \left(G_{q}^{+}(z)\right) \mathrm{d} z & =\operatorname{Res}\left[\frac{\mathrm{i} u}{z(z-\mathrm{i} u)} \log \left(G_{q}^{+}(z)\right) ; z=\mathrm{i} u\right] \\
& =\log G_{q}^{+}(\mathrm{i} u),
\end{aligned}
$$

where Res[.] is the residue of the corresponding pole. The first result in (3.10) follows because

$$
\int_{C_{R}^{+}} \frac{\mathrm{i} u}{z(z-\mathrm{i} u)} \log \left(G_{q}^{+}(z)\right) \mathrm{d} z \rightarrow 0, \quad R \rightarrow \infty,
$$

in view of (iii). The second result in (3.10) follows similarly. In fact, we have

$$
\int_{L_{R}} \frac{u}{z(z-\mathrm{i} u)} \log \left(G_{q}^{-}(z)\right) \mathrm{d} z=0,
$$

as the integrand is analytic in the interior of the contour, and

$$
\int_{C_{R}^{-}} \frac{\mathrm{i} u}{z(z-\mathrm{i} u)} \log \left(G_{q}^{+}(z)\right) \mathrm{d} z \rightarrow 0, \quad R \rightarrow \infty,
$$

also in view of (iii). This concludes the verification of (3.10) and the proof of the theorem for the case in which $q>0$.

The case in which $q=0$ is proved by approximation as follows. Observe that $(1 / q) \tau(1)$ has exponential distribution with parameter $q$ if $\tau(1)$ has exponential distribution with parameter 1. As $\tau(q) \rightarrow \infty$ almost surely (a.s.) when $q \rightarrow 0$, this shows that we can find exponential times such that $M_{q} \rightarrow M_{0}$ a.s. when $q \rightarrow 0$. For $q>0$, we have just obtained

$$
\mathrm{E}\left(\exp \left(-u M_{q}\right)\right)=\prod_{k=1}^{v}\left(\frac{u+\alpha_{k}}{\alpha_{k}}\right)^{n_{k}} \prod_{j=1}^{\mu(q)}\left(\frac{\beta_{j}(q)}{u+\beta_{j}(q)}\right)^{m_{j}(q)}=\frac{A_{n}(u)}{B_{m, q}(u)}
$$

We take limits as $q$ tends to 0 and apply (c) in Lemma 1.1 to obtain

$$
\mathrm{E}\left(\exp \left(-u M_{0}\right)\right)=\frac{A_{n}(u)}{B_{m, 0}(u)}
$$

concluding the proof of Theorem 2.2. 
Lemma 3.1. Consider a random variable $M \geq 0$ with distribution $F(x)$ such that

$$
\mathrm{P}(M>x) \sim A \mathrm{e}^{-\beta x}, \quad x \rightarrow \infty,
$$

for some $\beta>0$. Then its characteristic function

$$
\phi(z)=\int_{0}^{\infty} \mathrm{e}^{\mathrm{i} z x} F(\mathrm{~d} x)
$$

is analytic in the complex half-plane $\operatorname{Im}(z)>-\beta$. Moreover,

$$
\lim _{z \downarrow-\mathrm{i} \beta}\left(\frac{z+\mathrm{i} \beta}{\mathrm{i} \beta}\right) \phi(z)=A .
$$

Proof. The analyticity statement is a well-known consequence of the fact that $F(\mathrm{~d} x)$ is of exponential type. The statement about the pole is established in Proposition 3.1 of [16].

As a consequence of Lemma 3.1, the asymptotic constant $C$ in general Lévy models under the conditions of Proposition 2.1 is given by (3.11), which in our case reads exactly as in (2.13).

Proof of Proposition 2.1. The proof is based on Lemma 3.1.

\section{Acknowledgements}

Our ladder process discussion originated from a suggestion of one of the anonymous referees, both of whom made a number of helpful comments that improved an earlier version of this paper.

The second author thanks Paavo Salminen for hospitality at Åbo Akademi, Turku, Finland, where this article was partially written. He also thanks Mario Wschebor for helpful discussions. The second author was partially supported by CSIC-UDELAR, project I+D115.

\section{References}

[1] Asmussen, S. (1977). Ruin Probabilities (Adv. Ser. Statist. Sci. Appl. Prob. 2) World Scientific, Singapore.

[2] Asmussen, S., Avram, F. And Pistorius, M. (2004). Russian and American put options under exponential phase-type Lévy motion. Stoch. Process. Appl. 109, 79-111.

[3] Baxter, G. AND Donsker, M. D. (1957). On the distribution of the supremum functional for processes with stationary independent increments. Trans. Amer. Math. Soc. 85, 73-87.

[4] Bertoin, J. (1994). Lévy processes that can creep downwards never increase. Ann. Inst. H. Poincaré Prob. Statist. 31, 379-391.

[5] Bertoin, J. (1996). Lévy Processes (Cambridge Tracts Math. 121). Cambridge University Press.

[6] Bertoin, J. and Doney, R. (1994). Cramér's estimate for Lévy processes. Statist. Prob. Lett. 21, 363-365.

[7] Boyarchenko, S. I. And LevendorskiĬ, S. Z. (2002). Non-Gaussian Merton-Black-Scholes Theory (Adv. Ser. Statist. Sci. Appl. Prob. 9) World Scientific, Singapore.

[8] Chan, T. (2004). Some applications of Lévy processes in insurance and finance. Finance Rev. Assoc. Française Finance 25, 71-94.

[9] Doney, R. A. (1991). Hitting probabilities for spectrally positive Lévy processes. J. London Math. Soc. 44, $566-576$

[10] Feller, W. (1966). An Introduction to Probability Theory and Its Applications, Vol. II. John Wiley, New York.

[11] Furrer, H. (1998). Risk processes perturbed by $\alpha$-stable Lévy motion. Scand. Actuarial J. 1, 59-74.

[12] Harrison, J. M. (1977). The supremum distribution of a Lévy process with no negative jumps. Adv. Appl. Prob. 9, 417-422.

[13] HuZak, M., Perman, M., ŠIKić, H. AND VondračEK, Z. (2004). Ruin probabilities and decompositions for general perturbed risk processes. Ann. Appl. Prob. 14, 1378-1397.

[14] Kemperman, J. (1961). The Passage Problem for a Stationary Markov Chain. University of Chicago Press. 
[15] Klüppelberg, C., Kyprianou, A. E. and Maller, R. A. (2004). Ruin probabilities and overshoots for general Lévy insurance risk processes. Ann. Appl. Prob. 14, 1766-1801.

[16] KorevaAr, J. (2002). A century of complex Tauberian theory. Bull. Amer. Math. Soc. (N.S.) 39, 475-531.

[17] Kyprianou, A. E. (2006). Introductory Lectures on Fluctuations of Lévy Processes with Applications. Springer, Berlin.

[18] Mordecki, E. (2002). Optimal stopping and perpetual options for Lévy processes. Finance Stoch. 4, $473-493$.

[19] MordecKI, E. (2002). The distribution of the maximum of a Lévy process with positive jumps of phase-type. Theory Stoch. Process. 8, 309-316.

[20] Mordecki, E. (2003). Ruin probabilities for Lévy processes with mixed-exponential negative jumps. Theory Prob. Appl. 48, 170-176.

[21] Petrov, V. (1987). Limit Theorems for Sums of Independent Random Variables. Nauka, Moscow (in Russian).

[22] Pistorius, M. (2006). On maxima and ladder processes for a dense class of Lévy process. J. Appl. Prob. 43, 208-220.

[23] Rogers, L. C. G. (1984). A new identity for real Lévy processes. Ann. Inst. H. Poincaré Prob. Statist. 20, 21-34.

[24] Rogozin, B. (1966). On distributions of functionals related to boundary problems for processes with independent increments. Theory Prob. Appl. 11, 580-591.

[25] Sato, K.-I. (1999). Lévy Processes and Infinitely Divisible Distributions (Cambridge Studies Adv. Math. 68). Cambridge University Press.

[26] Vigon, V. (2002). Votre Lévy rampe-t-il? J. London Math. Soc. 65, 243-256.

[27] Zolotarev, V. M. (1964). The first passage time of a level and the behavior at infinity for a class of processes with independent increments. Theory Prob. Appl. 9, 653-662. 